\title{
Male carriage of Gardnerella vaginalis
}

\author{
S G DAWSON, * C A ISON, + G CSONKA, * AND C S F EASMON† \\ From the Departments of *Genitourinary Medicine and + Medical Microbiology, St Mary's Hospital \\ Medical School, London
}

SUMMARY The prevalence of Gardnerella vaginalis in the urethra of 430 men attending a clinic for sexually transmitted disease was $11 \cdot 4 \%$; it was significantly higher in heterosexuals $(14 \cdot 5 \%)$ than in homosexuals $(4 \cdot 5 \%)$. There was no evidence of rectal or subpreputial carriage of $G$ vaginalis, and urethral carriage was not associated with symptoms of urethritis.

\section{Introduction}

There is a close association between Gardnerella vaginalis and non-specific vaginitis. ${ }^{1}$ The disease can be established in women by the inoculation into the vagina of vaginal material from infected women ${ }^{1}$ or by the inoculation of pure cultures of $G$ vaginalis. $^{2}$ There is a high urethral carriage rate of gardnerellas among male partners of infected women, ${ }^{13}$ and many women whose sexual partners remain untreated become reinfected. ${ }^{1}$ These observations suggest that gardnerella-associated vaginitis is a sexually transmitted disease. Little is known, however, about the prevalence of $G$ vaginalis in the male population in general and whether it is associated with symptoms.

In this study we have determined the prevalence of urethral carriage of $G$ vaginalis in 430 men attending a clinic for sexually transmitted diseases, compared urethral carriage of $G$ vaginalis with subpreputial and anorectal carriage, and examined the association between carriage, symptoms of urethritis, and patterns of sexual activity.

\section{Patients and methods}

Urethral samples were taken from $\mathbf{4 3 0}$ men attending the Praed Street clinic for sexually transmitted diseases. In addition anorectal samples were taken from 57 of the $\mathbf{4 3 0}$ men and subpreputial samples from 105. Patients were chosen randomly with no attempt at selection on the basis of symptoms. None had received any antimicrobial treatment for at least one month.

Address for reprints: Dr C S F Easmon, Department of Bacteriology, Wright-Fleming Institute, St Mary's Hospital Medical School, London W2 1PG

Accepted for publication 7 March 1982

\section{CULTURE}

The urethral samples, collected with $1-\mu$ disposable inoculating loops, were cultured immediately on to human and horse blood $(5 \% \mathrm{v} / \mathrm{v})$ agar in doublechamber Petri dishes. This enabled any haemolysis produced on the two media to be compared directly. Inoculated plates were stored at $36^{\circ}$ in $7 \% \mathrm{CO}_{2}$ and transferred to the laboratory within four hours. Anorectal and subpreputial swabs were transported to the laboratory in Amies transport medium, where they were plated on to similar media supplemented with amphotericin B $2 \mathrm{mg} / \mathrm{l}$, gentamicin sulphate $4 \mathrm{mg} / \mathrm{l}$, and nalidixic acid $30 \mathrm{mg} / \mathrm{l}$. All plates were incubated at $36^{\circ}$ in $7 \% \mathrm{CO}_{2}$ for 48 hours.

Gardnerella vaginalis were identified as catalase and oxidase Gram-variable rods which produced a pronounced diffuse $\beta$-haemolysis on human, but little or no haemolysis on horse, blood agar and which fermented starch maltose and glucose. ${ }^{4}$

\section{Results}

Urethral samples were obtained from 430 men; $G$ vaginalis was isolated from 49 , giving a prevalence of $11 \cdot 4 \%$. Although quantitative cultures were not performed, a heavy growth of $G$ vaginalis was obtained in most cases. The double-chamber Petri dishes showed the differential $\beta$-haemolysis on horse and human agar very clearly (figure). There was a significant association between the isolation of $G$ vaginalis and the absence of symptoms of urethritis (table I). Subpreputial swabs from 105 men and rectal swabs from 57 showed as gardnerellas at either site, although $G$ vaginalis colonised the urethra of 13 and four men in the two groups.

The relationship between carriage of $G$ vaginalis and patterns of sexual behaviour is shown in table II. Carriage was significantly higher among heterosexuals $(14 \cdot 5 \%)$ than among homosexuals $(4 \cdot 5 \%)$. 


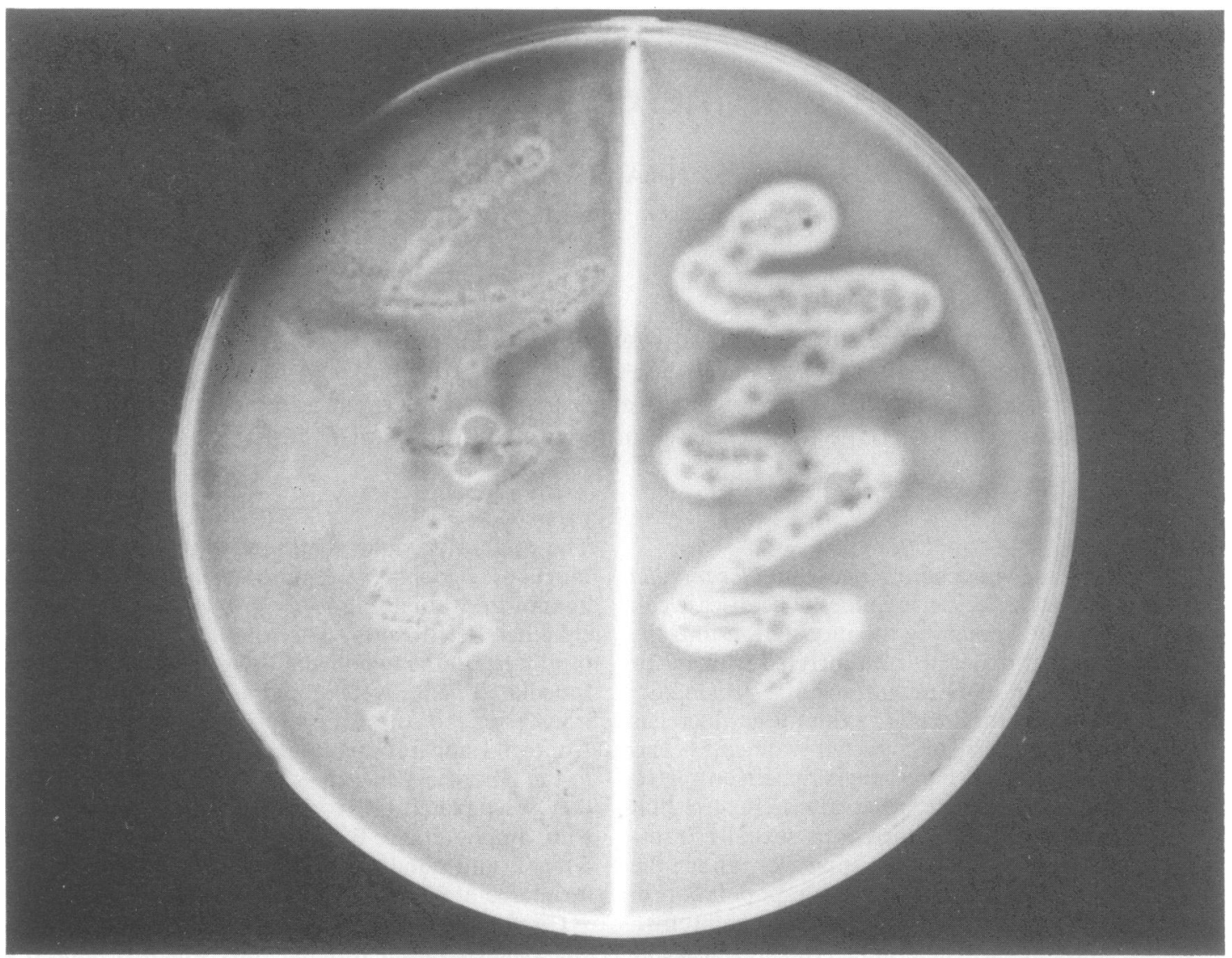

FIGURE Double-chamber Petri dish containing human blood agar on the right and horse blood agar on the left showing differential haemolysis produced by $G$ vaginalis.

TABLE I Relationship between symptoms of urethritis and urethral isolation of $G$ vaginalis

\begin{tabular}{lll}
\hline & \multicolumn{2}{c}{ Culture results for $G$ vaginalis (\% of total) } \\
\cline { 2 - 3 } Symptoms & Positive & Negative \\
\hline Present & $16(7 \cdot 2)^{*}$ & 206 \\
Absent & $33(15 \cdot 8)^{*}$ & 175 \\
\hline
\end{tabular}

${ }^{*} \chi^{2}=8 \cdot 0 ; 0 \cdot 01<P>0 \cdot 001$

TABLE II Urethral carriage of $G$ vaginalis in heterosexual, homosexual, and bisexual men

\begin{tabular}{lcc}
\hline & \multicolumn{2}{c}{ Culture results for $G$ vaginalis $(\%$ of total) } \\
\cline { 2 - 3 } & Positive & Negative \\
\hline Heterosexual & $42(14 \cdot 5)^{*}$ & 246 \\
Homosexual & $6(4 \cdot 5)^{*}$ & 127 \\
Bisexual & 1 & 8 \\
\hline
\end{tabular}

${ }^{*} \chi^{2}=9 \cdot 1 ; 0 \cdot 01<P>0 \cdot 001$

\section{Discussion}

Pheifer $e a^{3} l^{3}$ found $G$ vaginalis in the urethra of 27 of 34 male partners of patients with gardnerellaassociated vaginitis. Gardner and Dukes ${ }^{1}$ found the organism in 45 of 47 sexual contacts of women infected with $G$ vaginalis. Impressive though these figures are, neither study included comparable control groups. Our prevalence of $14.5 \%$ among an unselected population of sexually active male heterosexuals and the much lower prevalence among homosexuals does, if generally applicable, strongly support the idea of a male reservoir of $G$ vaginalis among the partners of infected women. We are now investigating carriage rates of $G$ vaginalis in this highly selected group.

The lack of any association between symptoms and urethral carriage of $G$ vaginalis suggests that it is not a primary pathogen. Gardner and Dukes ${ }^{1}$ showed that the organism could persist in the male urethra 
for many months. One case of cystitis apparently caused by $G$ vaginalis has been reported. ${ }^{5}$

We found no evidence of rectal carriage of $G$ vaginalis, although we have seen this in women. More surprising was our failure to isolate $G$ vaginalis from the subpreputial sac, even in those men who were urethral carriers. It is a fastidious organism, and this site may not provide the correct growth conditions for the persistence of $G$ vaginalis. Other organisms which colonise the male urethra, such as group B streptococci, can be isolated readily from both the anorectal area and the subpreputial sac.

Anaerobes have been implicated with $G$ vaginalis in the pathogenesis of non-specific vaginitis, ${ }^{3}$ and recently vibrio-like anaerobes have been isolated in such cases. ${ }^{6-8}$ Fontaine et $a l^{9}$ isolated curved anaerobic bacilli from men with non-gonococcal urethritis. We are investigating the relationship between gardnerellas and anaerobes in men and women.

We thank Dr J W R Harris for permission to study patients under his care. This work was supported by a grant from the Medical Research Council.

\section{References}

1. Gardner HL, Dukes CD. Haemophilus vaginalis vaginitis. A newly defined specific infection previously classified as "nonspecific vaginitis". Am J Obstet Gynecol 1955;69:962-76.

2. Criswell BS, Ladwig CL, Gardner HL, Dukes CD. Haemophilus vaginalis: vaginitis by inoculation from culture. Obstet Gynecol 1969; 33: 195-9.

3. Pheifer TA, Forsyth PS, Durfee MA, Pollock HM, Holmes KK. Non-specific vaginitis. Role of Haemophilus vaginalis and treatment with metronidazole. $N$ Engl J Med 1978; 298: 1429-34.

4. Ison CA, Dawson SG, Hilton J, Csonka G, Easmon CSF. A comparison of culture and microscopy in the diagnosis of Gardnerella vaginalis infection. J Clin Pathol 1982 (in press).

5. Birch DF, D'Apice AJF, Fairley KF. Ureaplasma urealyticum in the upper tracts of renal allograft recipients. $J$ Infect Dis 1981;144:123-7.

6. Hjelm E, Hallén A, Forsum U, Wallin J. Anaerobic curved rods in vaginitis. Lancet 1981 ; ii: $1353-4$.

7. Sprott MS, Pattman RS, Ingham HR, Short GR, Narang HK, Selkon JB. Anaerobic curved rods in vaginitis. Lancet 1982 i: 54 .

8. Phillips I, Taylor E. Anaerobic curved rods in vaginitis. Lancet 1982; i: 221 .

9. Fontaine EA, Taylor-Robinson D, Borriello SP, Hanna NF Honour P. Anaerobic bacteria in lower genital tract infections. Lancet 1982; i: 281. 